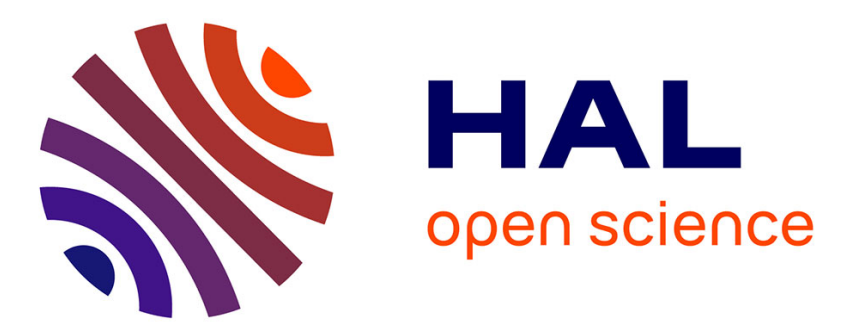

\title{
Design and Evaluation of a Wearable Skin Stretch Device for Haptic Guidance
}

Francesco Chinello, Claudio Pacchierotti, Joao Bimbo, Nikos G Tsagarakis, Domenico Prattichizzo

\section{- To cite this version:}

Francesco Chinello, Claudio Pacchierotti, Joao Bimbo, Nikos G Tsagarakis, Domenico Prattichizzo. Design and Evaluation of a Wearable Skin Stretch Device for Haptic Guidance. IEEE Robotics and Automation Letters, 2018, 3 (1), pp.524 - 531. 10.1109/LRA.2017.2766244 . hal-01935192

\section{HAL Id: hal-01935192 \\ https://hal.inria.fr/hal-01935192}

Submitted on 26 Nov 2018

HAL is a multi-disciplinary open access archive for the deposit and dissemination of scientific research documents, whether they are published or not. The documents may come from teaching and research institutions in France or abroad, or from public or private research centers.
L'archive ouverte pluridisciplinaire $\mathbf{H A L}$, est destinée au dépôt et à la diffusion de documents scientifiques de niveau recherche, publiés ou non, émanant des établissements d'enseignement et de recherche français ou étrangers, des laboratoires publics ou privés. 


\title{
Design and evaluation of a wearable skin stretch device for haptic guidance
}

\author{
Francesco Chinello, Claudio Pacchierotti, Member, IEEE, Joao Bimbo, Member, IEEE, \\ Nikos G. Tsagarakis, Senior Member, IEEE, Domenico Prattichizzo, Fellow, IEEE
}

\begin{abstract}
We present a wearable skin stretch device for the forearm. It is composed of four cylindrical end effectors, evenly distributed around the user's forearm. They can generate independent skin stretch stimuli at the palmar, dorsal, ulnar, and radial sides of the arm. When the four end effectors rotate in the same direction, the wearable device provides cutaneous stimuli about a desired pronation/supination of the forearm. On the other hand, when two opposite end effectors rotate in different directions, the cutaneous device provides cutaneous stimuli about a desired translation of the forearm. To evaluate the effectiveness of our device in providing navigation information, we carried out two experiments of haptic navigation. In the first one, subjects were asked to translate and rotate the forearm toward a target position and orientation, respectively. In the second experiment, subjects were asked to control a 6-DoF robotic manipulator to grasp and lift a target object. Haptic feedback provided by our wearable device improved the performance of both experiments with respect to providing no haptic feedback. Moreover, it showed similar performance with respect to sensory substitution via visual feedback, without overloading the visual channel.
\end{abstract}

\section{INTRODUCTION}

Haptic devices capable of providing only cutaneous stimuli have recently gained a great deal of attention in the haptics and robotics research fields. Cutaneous feedback provides an effective way to simplify the design of haptic interfaces [1], [2], and it has been proven to play a key role in enhancing the performance and effectiveness of telepresence and immersive systems [3]-[5]. It is also particularly promising for robotic teleoperation applications, as cutaneous stimuli do not affect the stability and safety of the control loop [3], [5]-[8], and navigation applications, as cutaneous stimuli can provide rich information in an unobtrusive and private way.

Indeed, several researchers have developed and used cutaneous devices for navigation purposes. Spiers et al. [9] designed a shape-changing handheld device to provide pedestrian navigation instructions. The device resembled a cube with an upper half that rotates and translates with

F. Chinello is with the Dept. Business Development and Technology at Aarhus University in Herning, Midtjylland, Denmark. E-mail: chinello@btech.au.dk

C. Pacchierotti is with the CNRS at Irisa and Inria Rennes Bretagne Atlantique, Rennes, France. E-mail: claudio.pacchierotti@irisa.fr.

J. Bimbo, N. G. Tsagarakis, and D. Prattichizzo are with the Dept. Advanced Robotics, Fondazione Istituto Italiano di Tecnologia, Genova, Italy. E-mail: \{nikos.tsagarakis, domenico.prattichizzo\}@iit.it

D. Prattichizzo is also with the Dept. Information Engineering and Mathematics, University of Siena, Siena, Italy.

This research received funding from the EU Seventh Framework Programme FP7/2007-2013 under grant $n^{\circ} 601165$ of the project "WEARHAP WEARable HAPtics for humans and robots," and from Rennes Métropole under the "Allocation d'Installation Scientifique (AIS) 2017" programme.

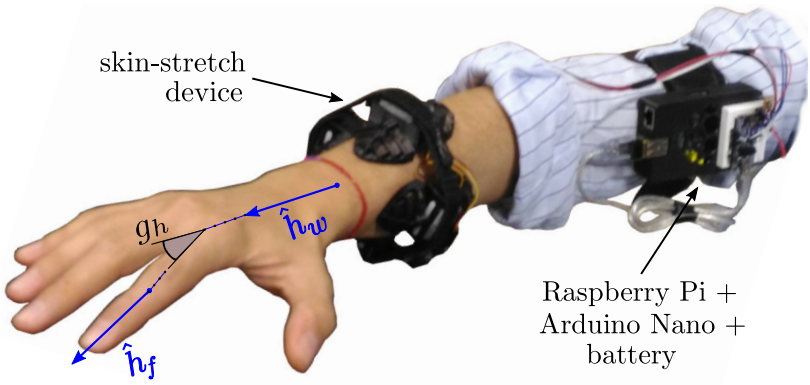

(a) View of the wearable skin stretch cutaneous device.

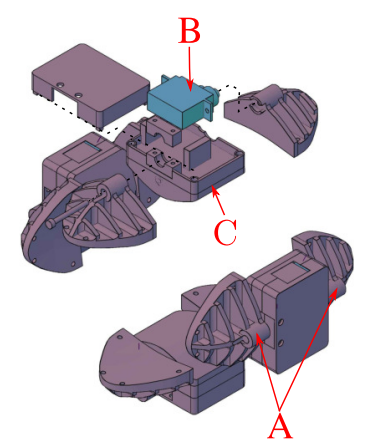

(b) CAD design.

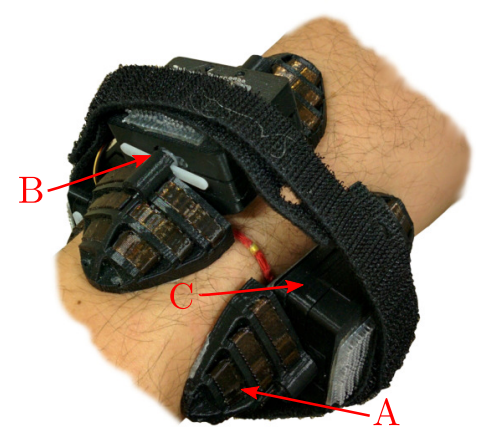

(c) Device being worn.
Fig. 1. Prototype of the wearable skin stretch cutaneous device. Four cylindrical end effectors provide independent skin stretch stimuli at the palmar, dorsal, ulnar, and radial sides of the arm. It is composed of the four cylindrical end effectors (C), that accommodate four servomotors (B), and eight ergonomic pads (A), one in the rear and one in the front of each end effector.

respect to the bottom half, that is grounded in the user's hand when held. The pose actuated by the device's upper half corresponds to heading and proximity to the target. Similarly, Imamura et al. [10] described a shape-changing handrail to guide users in unknown environments, and Hemmert [11] presented a shape-changing mobile phone that moves its back cover to point the user toward the target. In addition to shape-changing devices, there is also an increasing interest in vibrotactile cutaneous feedback. The compact and lightweight form factor of vibrotactile motors has in fact enabled researchers to design highly wearable and portable vibrotactile interfaces. For example, Oron-Gilad [12] used two vibrotactile motors on the hand to convey directional information. The motors were placed either on the palm or on the back of the hand. Results found no differences between the two configurations. However, placing the motors on the back of the hand was preferred to avoid any impairment when 


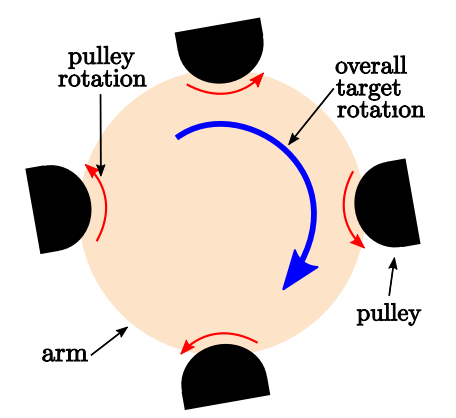

(a) Guidance for clockwise rotation (pronation)

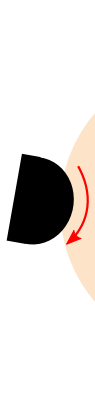

(b) Guidance for counter-clockwise rotation (supination)

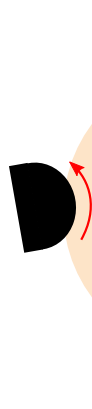

(c) Vertical motion of the wrist (dorsal motion)

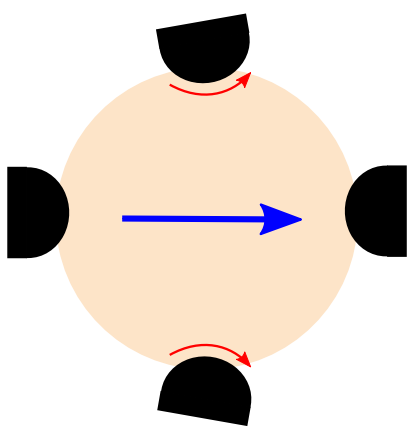

(d) Horizontal motion of the wrist (ulnar motion)

Fig. 2. Working principle. When the four end effectors rotate in the same direction, the wearable device conveys cutaneous feedback about a desired pronation/supination of the forearm (see (a) and (b)). On the other hand, when two opposite end effectors rotate in opposite directions, the device conveys cutaneous feedback about a desired translation of the forearm (see (c) and (d)).

grasping objects. More recently, a similar force illusion has been used by Rekimoto [13] for pedestrian guiding systems.

Another group of cutaneous devices providing navigation information uses skin stretch stimuli. These devices exploit the high sensitivity of human skin to tangential stretches and can provide the human user with rich directional information. Gleeson et al. [14], for example, introduced a two-degreesof-freedom (2-DoF) skin stretch device for the fingertip. It laterally stretches the finger pulp using a $7 \mathrm{~mm}$ hemispherical tactor. Two RC servo motors and a compliant flexure stage can move the tactor in the plane of the finger pad.

However, the hand is not the only place where we can provide effective haptic guidance stimuli. One of the main reasons to move away from the hand is to enable a more direct and natural interaction with the surrounding environment. For example, this is particularly relevant in haptic-enabled Augmented Reality scenarios [15], where users are asked to interact with virtual and real objects mixed together. But this is also relevant for navigation applications, where the aim is to provide effective and private navigation information to the users without impairing their motion and capability to interact with the environment. For these reasons, even though the hand is often regarded as a good location to convey haptic stimuli, it is common to find cutaneous devices that provide stimuli to other parts of the body. In this respect, the forearm has been often deemed as an effective placement to provide haptic guidance without impairing the user's hand. For example, Stanley and Kuchenbecker [16] tested five different cutaneous devices in wrist rotation guidance tasks. They were able to provide ten different forms of tactile feedback. Repeatedly tapping on the users' wrist on the side towards which they should turn showed the best performance. Rotella et al. [17] developed five haptic bands to be worn on the arms and wrists. They were instrumented with eccentric mass motors providing vibrotactile feedback for guidance of static poses. More recently, Yem et al. [18] presented a haptic display for hand guidance. This wearable outer-covering device is composed of two spherical end effectors that provide guidance information about the wrist's rotation and translation.

In this work, we present a novel cutaneous haptic device that provides navigation cues to the forearm in the form of lateral skin stretch haptic feedback, shown in Figs. 1. Four cylindrical rotating end effectors, located on the user's forearm, can generate skin stretch at the ulnar, radial, palmar, and dorsal sides of the arm. When all the end effectors rotate in the same direction, the cutaneous device is able to provide cues about a desired pronation/supination of the forearm (see Figs. 2a and 2b). On the other hand, when two opposite end effectors rotate in opposite directions, the device is able to provide cutaneous cues about a desired translation of the forearm (see Figs. 2c and 2d). Combining these two stimuli, we can provide both rotation and translation guidance to the forearm. With respect to other systems presented in the literature [17]-[20], this device is extremely wearable and unobtrusive [1]. Moreover, with respect to [9], [19], [21], it does not impair the hand. It weights $95 \mathrm{~g}$, it adds $2.5 \mathrm{~cm}$ to the wrist size, and it is wireless. Moreover, with respect to other forearm solutions [16], [20], [22], it can provide information about both the rotation and translation of the wrist. A separate arm band, weighting $280 \mathrm{~g}$, provides the required power and electronics. A preliminary version of this device has been presented in [23].

\section{THE WEARABLE SKIN STRETCH DEVICE}

The proposed wearable skin stretch cutaneous device is sketched in Fig. 1b, while a prototype is shown in Figs. 1a and 1c. It is composed of four cylindrical end effectors (indicated as "C" in Figs. 1b and 1c), that accommodate four Hitec HS-40 servomotors (B), and eight ergonomic pads (A), one in the rear and one in the front of each end effector. The end effectors and the ergonomic pads are made of ABSPlus. To improve grip and reduce slippage while contacting the skin, the end effectors are covered with rubber. The bracelet is wired to an arm band on the upper arm, that hosts a Raspberry Pi Model B, an Arduino Mini, and two 2 Ah batteries. The arm band is in charge of providing the required power to the device and manage the wireless communication between the device and an external computer. The cutaneous device weights $95 \mathrm{~g}$ while the arm band equipped with the batteries and control system weights $280 \mathrm{~g}$. 


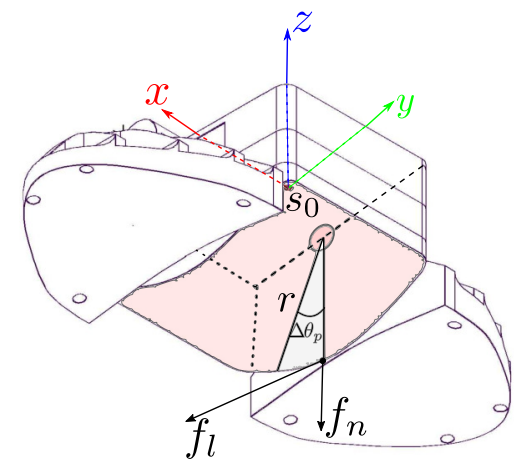

Fig. 3. Kinematic scheme of one skin stretch module. The proposed wearable device is composed of four skin stretch modules.

If no slip occurs, the movement of one of the end effectors onto the skin $\overline{\Delta S}$ directly results in the same amount of skin stretch. This movement can be calculated as

$$
\overline{\Delta S}=\Delta \theta_{p} \cdot r
$$

where $r=20 \mathrm{~mm}$ is the end effector radius (see Fig. 3), and $\Delta \theta_{p}$ is the commanded angle motion in radians.

To maximize the comfort and effectiveness of the haptic device, we placed it $10 \mathrm{~cm}$ proximal to the lunate bone (i.e., near the wrist) and fasten it until the end effector applied a force of $4 \mathrm{~N}$ normal to the skin. More details about this choice can be found in [23].

\section{EXPERIMENTAL EVALUATION}

To evaluate the effectiveness of our device in providing informative skin stretch sensations, we carried out two experiments of haptic navigation.

\section{A. Experiment \#1: preliminary arm guidance}

In the first experiment, we aim at evaluating the guiding capabilities of our device in a simple wrist navigation task.

1) Experimental setup and task: The experimental setup is composed of our wearable skin stretch device, as shown in Fig. 1. The force applied on the forearm skin was registered at the beginning of the experiment using four piezoresistive Force Sensing Resistor (FSR) sensors placed between the end effectors and the forearm. The FSR sensors were then removed to enable the subject to carry out the tasks. At the end of the experiment, the sensors were re-positioned to register any significant change in the fastening force. The maximum reduction of fastening force we registered was $0.6 \mathrm{~N}$, which we found acceptable. No subject reported any significant change in the fastening force or in the positioning of the device.

To detect pronation, supination, and translation of the forearm, we used a Leap Motion tracker.

The task consisted of rotating or translating the forearm following the navigation cues provided by the skin stretch device, being as accurate as possible. A video of the experiment is available as supplemental material. To avoid external distractions, subjects wore noise-canceling headphones and were blindfolded. Reference rotations and translations were

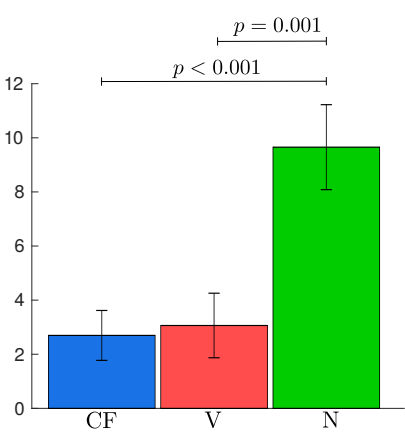

(a) Rotation error (degrees)

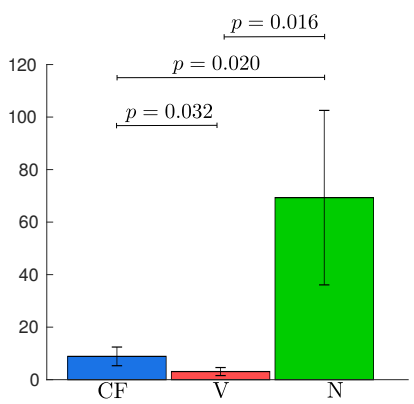

(b) Translation error (mm)
Fig. 4. Experiment \#1. Subjects were asked to rotate/translate the forearm following the navigation information provided by the device, being as accurate as possible. Absolute orientation and position error provided a measure of performance. Mean and $95 \%$ confidence interval are plotted.

uniformly chosen in the $\theta_{r} \in[10,60]^{\circ}$ and $d_{r} \in[1,10] \mathrm{cm}$ ranges, respectively. The Leap Motion tracked the position and orientation of the forearm. The task started when the subject moved the forearm for the very first time and ended when the subject felt to be in the right position.

2) Subjects and methods: Ten participants were involved in this first experiment, including three women and seven men. Seven participants had previous experience with haptic interfaces. Before starting the task, the experimenter explained the procedure and spent about two minutes adjusting the setup to be comfortable. One practice trial was allowed.

Each subject carried out thirty-six randomized trials of the navigation task, with twelve repetitions for each feedback condition:

- navigation feedback regarding the desired rotation/translation of the forearm using our wearable skin stretch device (condition $\mathrm{CF}$ ),

- navigation feedback regarding the desired rotation/translation of the forearm using visual cues (condition $\mathrm{V}$ ),

- no navigation feedback (condition $\mathrm{N}$ ),

In the first condition $\mathrm{CF}$, the wearable device provides haptic navigation feedback about pronation, supination, and translation of the forearm, as detailed in Sec. II and shown in Fig. 2. Subjects should thus rotate/translate their forearm following the cutaneous information provided by the device. The closer the subject is to the target orientation/position of the forearm, the less stretch the device applies to the skin. When the subject reaches the desired orientation/position, the device applies no skin stretch to the forearm. Another viable control policy would have been to directly provide the target hand pose, so that subjects would have had to try maintaining a constant skin stretch. However, applying a constant stretch stimulus for a long period results in a reduced sensation [24]. For this reason, we decided to provide information about the error between the current and target hand poses. To avoid slippage and achieve the highest accuracy, the cutaneous device maps the target rotation/translation to a skin stretch in the $[-8.75,8.75] \mathrm{mm}$ range, corresponding to a pulley rotation in the $\theta_{p} \in[-25,25]^{\circ}$ range [23]. This proportional control 
policy for controlling the device rotations and translation during the experiment is summarized by the two algorithms below.
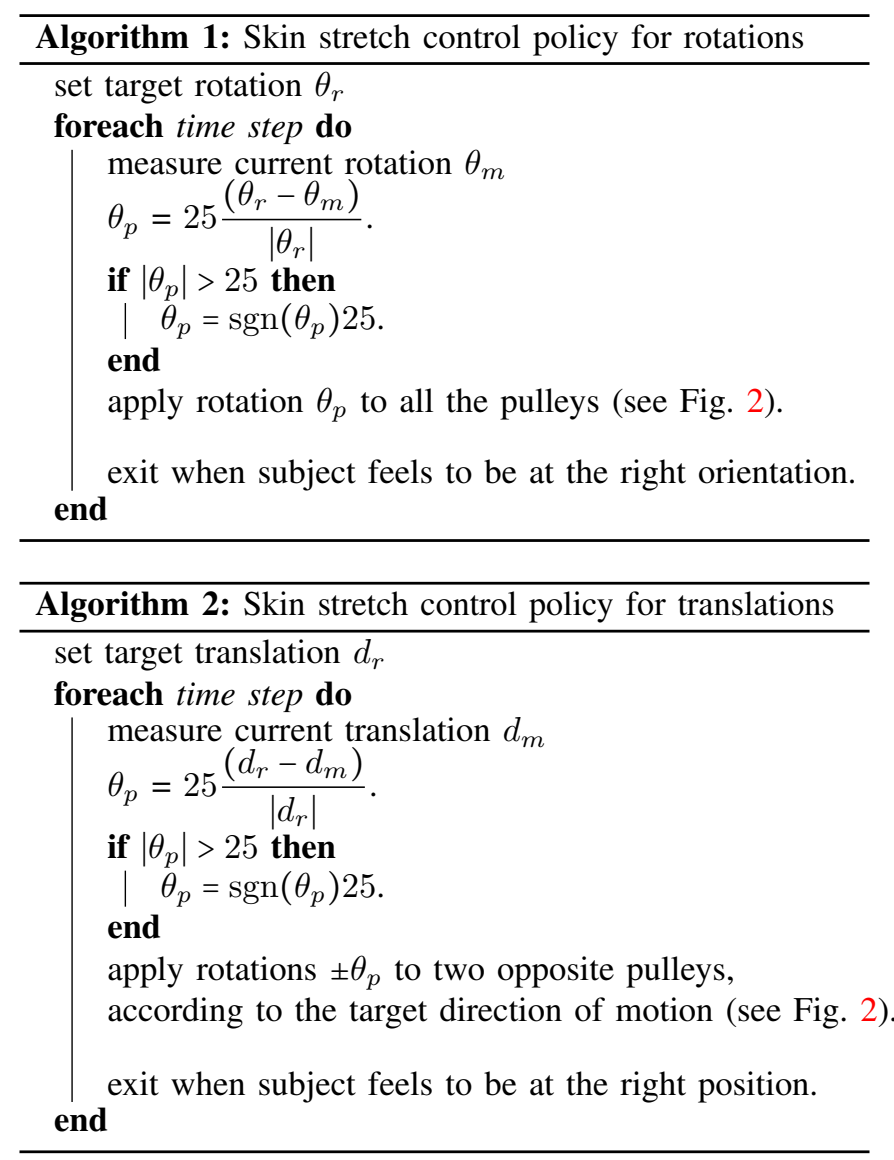

In condition $\mathrm{V}$, no cutaneous feedback is provided to the subjects. An LCD screen is placed in front of them and an arrow indicates the target direction or orientation. The length of the arrow indicates how far the subject is from the target direction or orientation. In condition $\mathrm{N}$, neither cutaneous nor visual feedback is provided to the subjects. At the beginning of the task, the experimenter communicates the target movement to the subject.

The ten participants carried out twelve repetitions of the navigation task in each feedback condition, two times for each navigation modality: pronation (Fig. 2a), supination (Fig. 2b), translation to the right (Fig. 2d), translation to the left, elevation (Fig. 2c), and lowering of the forearm.

3) Results: Rotation and position errors as well as completion time provided a measure of performance. Low values for these metrics indicate the best performance.

Fig. 4a shows the average absolute rotation error at the end of the task. All the data passed the Shapiro-Wilk normality test and Mauchly's Test of Sphericity. A one-way repeatedmeasures ANOVA test showed statistically significant changes between the feedback conditions $(\mathrm{F}(2,18)=35.351, \mathrm{p}<$ 0.001). Post-hoc analysis with Bonferroni correction showed statistically significant differences between $\mathrm{CF}$ vs. $\mathrm{N}$ with $\mathrm{p}<0.001$ and $\mathrm{V}$ vs. $\mathrm{N}$ with $\mathrm{p}=0.001$. Fig. 4b shows the average absolute translation error at the end of the task for each condition. All the data passed the Shapiro-Wilk normality test. Mauchly's Test of Sphericity indicated that the assumption of sphericity was violated $\left(\chi^{2}=22.286, p<\right.$ 0.001). A one-way repeated-measures ANOVA test with a Greenhouse-Geisser correction showed statistically significant changes between the feedback conditions $(\mathrm{F}(1.016,9.141)=$ $13.267, \mathrm{p}<0.001)$. The Greenhouse-Geisser correction is used when the assumptions of sphericity or equal variances are violated, as in this case. It corrects the analysis by calculating new degrees of freedom and a new significance value $p$, so that a valid F-ratio can be obtained. Post-hoc analysis with Bonferroni correction showed statistically significant differences between all conditions: $\mathrm{CF}$ vs. V, $\mathrm{p}=0.032 ; \mathrm{CF}$ vs. $\mathrm{N}, \mathrm{p}=0.020$; and V vs. $\mathrm{N}, \mathrm{p}=0.016$. Finally, we also measured the completion time. All the data passed the ShapiroWilk normality test and Mauchly's Test of Sphericity. A one-way repeated-measures ANOVA indicated no significant difference in the performance among feedback conditions. Average completion time was $5.06 \pm 1.02 \mathrm{~s}$ (mean $\pm \mathrm{SD}$ ). Immediately after the experiment, subjects were also asked to choose the condition they preferred the most. Condition $\mathrm{CF}$ was preferred by two subjects, while condition $\mathrm{V}$ was preferred by eight subjects.

\section{B. Experiment \#2: robotic telemanipulation}

In the second experiment, we aim at evaluating the guiding capabilities of our device in a robotic telemanipulation task.

1) Experimental setup and task: The experimental setup and remote environment are shown in Fig. 5. The master side is composed of our skin stretch device, as shown in Fig. 1. Moreover, a Leap Motion tracker is in charge of registering the position, orientation, and grasping configuration of the operator's hand. The hand grasping configuration, i.e., how closed the hand is, is measured by considering the radian angle $g_{h}$ between the index finger's distal phalanx direction $\hat{h}_{f}$ and the hand direction $\hat{h}_{w}$ (see Fig. 1a and [25]),

$$
g_{h}=\cos ^{-1}\left(\hat{h}_{f} \cdot \hat{h}_{w}\right) \text {. }
$$

The slave robot is composed of the anthropomorphic Pisa/IIT SoftHand soft robotic hand, attached to a 6-DoF Universal Robot 5 manipulator (Universal Robots A/S, Denmark). A small camera is attached to the robot's wrist, showing the environment in front of the robotic hand. The SoftHand uses only one actuator to activate its adaptive synergy [26]. The index, middle, ring, and little fingers have four phalanges, while the thumb has three. The hand is connected to a flange, to be fixed at the manipulator's end effector, through a compliant wrist allowing for three passively compliant degrees of freedom. The motor of the hand is driven by commanding a grasping variable $g_{r} \in[0,1]$, which sets the hand closure. The 6-DoF manipulator is able to move the end effector at up to $1 \mathrm{~m} / \mathrm{s}$ and each joint at $180 \mathrm{deg} / \mathrm{s}$. Its repeatability is $\pm 0.1 \mathrm{~mm}$ for quick-precision handling with a maximum payload of $5 \mathrm{~kg} \mathrm{[27].}$

The desired pose of the robotic hand and wrist at time $t$, ${ }^{B} \boldsymbol{T}_{D}(t)$, is commanded using a mapping between the SoftHand and the user's hand, tracked by the Leap Motion [25]. 


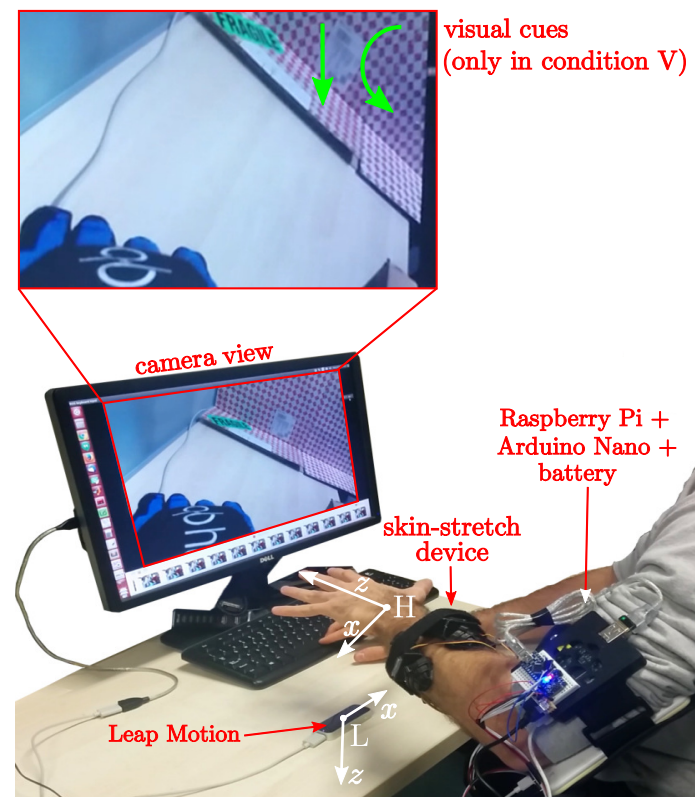

(a) Master side.

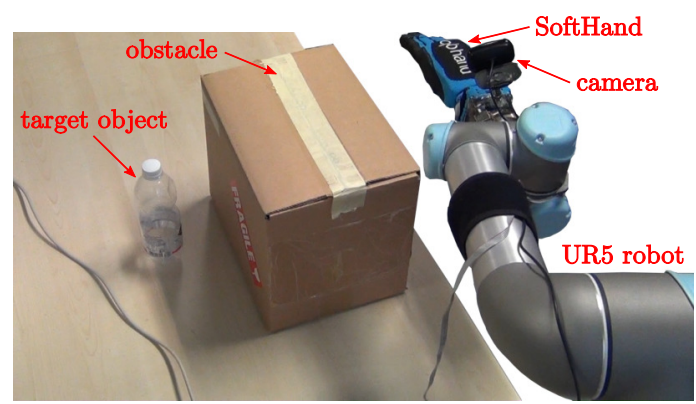

(b) Slave side.

Fig. 5. Experiment \#2. The master system is composed of the wearable skin stretch device and a Leap Motion controller. The slave system is composed of the Pisa/IIT SoftHand soft robotic hand attached to the end effector of a 6-DoF Universal Robot 5 manipulator. A camera attached to the robot's wrist shows the remote environment to the human operator. The desired pose and grasping configuration of the robotic hand are controlled by the human operator as detailed in Sec. III-B.1. The keyboard works as a clutch.

The keyboard works as a clutch. All coordinate frames but the one on the robot base $B$ are shown in Figs. 5 and 6 . Frame $B$ has its origin on the robot base, with the $z$ axis orthogonal to the table (pointing up), the $x$ axis parallel to the table (pointing toward the obstacle), and the $y$ consequently defined. When the space key is pressed, the current pose of the user's hand with respect to the Leap Motion base ${ }^{L} \boldsymbol{T}_{H} \in \mathrm{SE}(3)$ and the robot's end effector pose with respect to its base ${ }^{B} \boldsymbol{T}_{E}$ are saved:

$$
\begin{aligned}
& { }^{L} \boldsymbol{T}_{H}(0)={ }^{L} \boldsymbol{T}_{H}(t) \\
& { }^{B} \boldsymbol{T}_{E}(0)={ }^{B} \boldsymbol{T}_{E}(t) .
\end{aligned}
$$

As the user moves his hand, the desired end effector pose is calculated according to eq. (3), using the poses saved when the user started pressing the clutch, such that their relative motions are equal, i.e., ${ }^{E_{0}} \boldsymbol{T}_{D}={ }^{H_{0}} \boldsymbol{T}_{H}$,

$$
{ }^{B} \boldsymbol{T}_{D}(t)={ }^{B} \boldsymbol{T}_{E}(0) \cdot{ }^{L} \boldsymbol{T}_{H}^{-1}(0) \cdot{ }^{L} \boldsymbol{T}_{H}(t) .
$$

This mapping allows the robot to be commanded in a natural way while looking at the camera: if the user moves his hand in the $z$ direction of the $H$ frame, the robot will move along the $z$ direction of its frame $E$. This approach also allows the user to start the motion with the hand at an arbitrary position with respect to the Leap Motion, enabling the user to also pause, move to a more comfortable or suitable position, and then resume the control of the robot.

To also match as closely as possible the grasping configuration of the operator's hand, we define the input commanded to the hand DC motor as $g_{r}=g_{h} \pi^{-1}$, where a command of $0 \%$ closure is sent when the index is parallel with the palm direction and $100 \%$ closure is commanded when the index finger is bent $180^{\circ}$ (see eq. (2) and Fig. 1a).

The remote environment is composed of the slave robot, an obstacle, and a target object to grasp (i.e., a bottle of water). The task consisted of moving the slave robot in the remote environment and picking up the target object, following the navigation information provided by the device and avoiding contacts with obstacles (see Fig. 5). A video of the experiment is available as supplemental material.

2) Subjects and Methods: Ten participants took part in the experiment, including one women and nine men. Six of them had previous experience with haptic interfaces. Before starting the experiment, the experimenter explained the procedure and spent about three minutes adjusting the setup to be comfortable. One practice trial was allowed.

We considered two different trajectories $\left(t_{r}\right.$ vs. $\left.t_{p}\right)$, two different orientations of the target object to grasp $\left(\mathrm{o}_{d}\right.$ vs. $\left.\mathrm{o}_{u}\right)$, and three different haptic feedback conditions (CF vs. V vs. $\mathrm{N}$ ), ending up with 2 (trajectories) $\times 2$ (object orientations) $\times$ 3 (feedbacks) $=12$ different experimental conditions. The two trajectories are shown in Fig. 6. The first trajectory moves the robot following a rectangular shape around the obstacle $\left(t_{r}\right)$, while the second one moves the robot following a parabolic shape around the obstacle $\left(\mathrm{t}_{p}\right)$. The water bottle to grasp can be either standing $\left(\mathrm{o}_{u}\right)$ or laying $\left(\mathrm{o}_{d}\right)$ on the table. The configuration of the bottle on the table also defines the final target orientation for the robotic hand with respect to the $B$ frame, chosen to enable a successful grasp: $\gamma=\operatorname{rot}(x)_{B}=0^{\circ}$ for $\mathrm{o}_{d}$ (hand palm parallel to the table) and $\gamma=\operatorname{rot}(x)_{B}=90^{\circ}$ for $\mathrm{o}_{u}$ (hand palm perpendicular to the table), i.e., with the palm parallel to the bottle. The starting position and orientation of the hand are chosen randomly around the first point of the trajectory, as shown in Fig. 6. Finally, the human operator receives navigation feedback about the desired trajectory and orientation through our skin stretch device (CF), through sensory substitution via visual feedback $(\mathrm{V})$, or receive no feedback at all $(\mathrm{N})$.

Each trajectory is divided into 60 equidistant points (green squares in Fig. 6). Given the hand position, the current target point is defined as the point along the trajectory which comes next after the one closest to the robotic hand (red square in Fig. 6). All the points except the last one commands a target orientation of $\operatorname{rot}(x)_{B}=0$. The target orientation for the last point depends on the bottle configuration, as specified above. The control policy for this experiment is summarized in the 


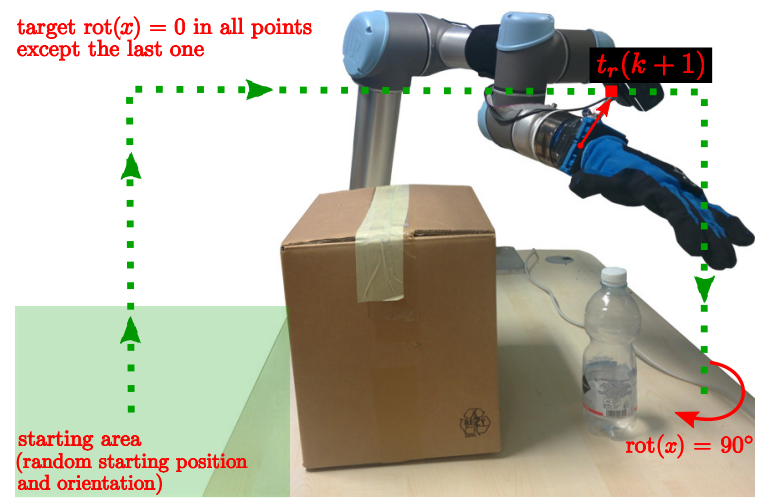

(a) Trajectory $\mathrm{t}_{r}$ with the bottle standing on the table $\left(\mathrm{o}_{u}\right)$

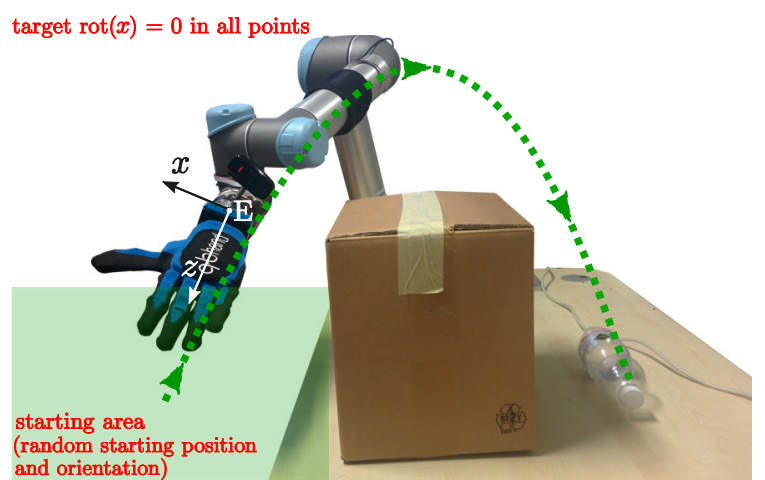

(b) Trajectory $\mathrm{t}_{p}$ with the bottle laying on the table $\left(\mathrm{o}_{d}\right)$

Fig. 6. Experiment \#2. The task consisted of moving the slave robot in the remote environment and picking up the plastic bottle, following the navigation information provided by the device and avoiding contacts with obstacles. A video of the experiment is available as supplemental material.

algorithm reported below.

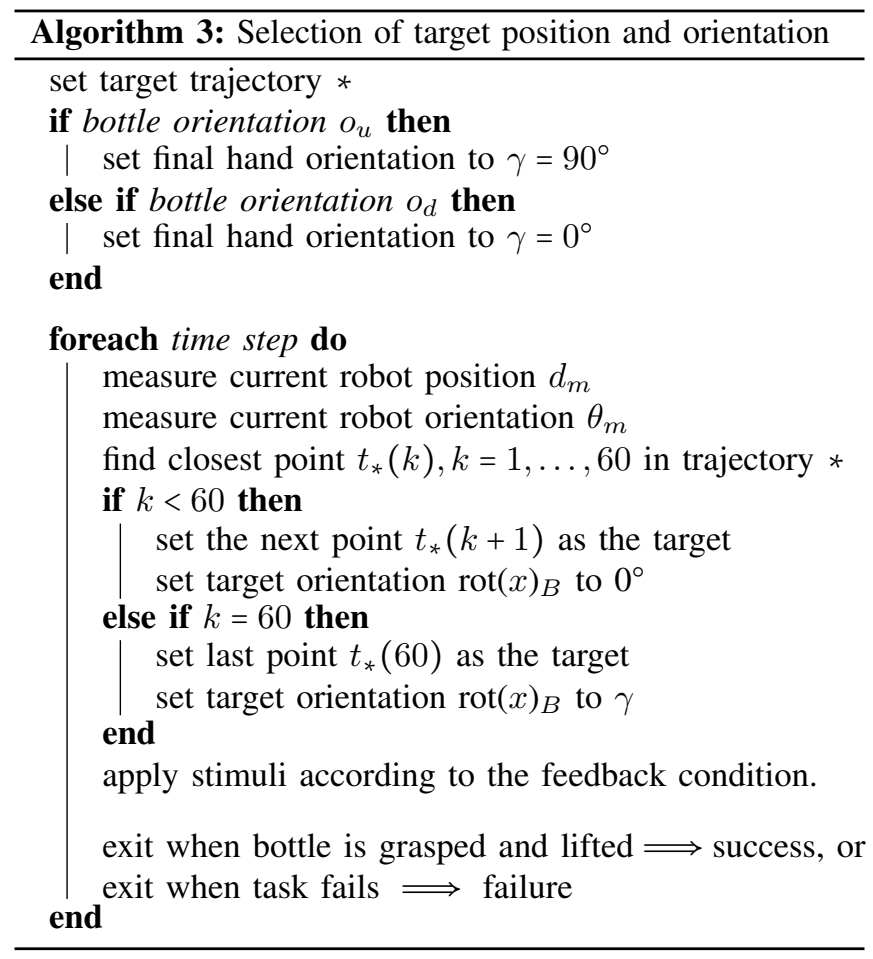

In condition $\mathrm{CF}$, our wearable cutaneous device provides the subject with navigation information about the translation and orientation of the robotic hand. Similarly to Sec. III-A, the more the subject rotates/translates the forearm toward the target point of the trajectory, the less stretch the device applies to the skin. As before, to avoid slippage and provide the highest accuracy, the cutaneous device always maps the target rotation/translation to a skin stretch in the range $[-8.75,8.75] \mathrm{mm}$. Once the target position and orientation is defined as per Algorithm 3, skin stretch stimuli are provided to the human operator combining the control policies detailed in Algorithms 1 and 2. In condition V, no cutaneous feedback is provided to the subjects. An arrow, superimposed on the camera view of the remote environment, indicates the target direction and orientation of the robotic hand. As for Sec. III-
A, the length of the arrow indicates how far the subject is from the target direction or orientation. In condition N, no navigation feedback is provided to the subjects. Before the beginning of the task, the experimenter shows the subject a picture of the target trajectory and orientation superimposed to the environment, e.g., Fig. 6a or Fig. 6b. The operator has then to rely only on the visual information provided by the camera to complete the grasping task. Subjects performed twenty-four repetitions of the navigation task, two for each experimental condition.

3) Results: Translation error, orientation error, and outcome of the task were measures of performance.

Fig. 7a shows the average absolute orientation error, calculated as the RMS of the difference in orientation between the robotic hand and the target orientation during the task. All the data passed the Shapiro-Wilk normality test and Mauchly's Test of Sphericity. A one-way repeated-measures ANOVA test revealed statistically significant changes between the feedback conditions $(\mathrm{F}(2,18)=24.408, \mathrm{p}<0.001)$. Posthoc analysis with Bonferroni correction revealed statistically significant difference between $\mathrm{CF}$ vs. $\mathrm{N}$ with $\mathrm{p}=0.003$ and $\mathrm{V}$ vs. $\mathrm{N}$ with $\mathrm{p}=0.001$. Fig. $7 \mathrm{~b}$ shows the average absolute translation error, calculated as the RMS of the distance between the robotic hand and the target trajectory during the task. All the data passed the Shapiro-Wilk normality test. Mauchly's Test of Sphericity indicated that the assumption of sphericity had been violated $\left(\chi^{2}=22.450, p<0.001\right)$. A one-way repeated-measures ANOVA test with a GreenhouseGeisser correction revealed statistically significant changes between the feedback conditions $(\mathrm{F}(1.031,9.280)=25.387$, $\mathrm{p}=0.001$ ). Post-hoc analysis with Bonferroni correction revealed statistically significant difference between conditions CF vs. $\mathrm{N}, \mathrm{p}=0.002$; and $\mathrm{V}$ vs. $\mathrm{N}, \mathrm{p}=0.002$. Fig. $7 \mathrm{c}$ shows the average success rate of the task. A task was considered successfully completed when the slave robot was able to grasp the bottle and lift it from the ground. A Friedman test showed a statistically significant difference between the means of the feedback conditions $\left(\chi^{2}(2)=8.909, p=0.012\right)$. Post hoc analysis with Bonferroni correction revealed a statistically significant difference between conditions CF vs. $\mathrm{N}(\mathrm{p}=0.002)$ 


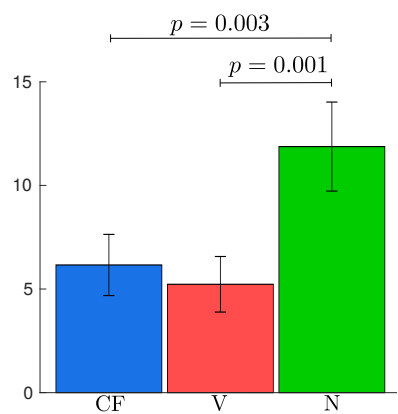

(a) Rotation error (degrees)

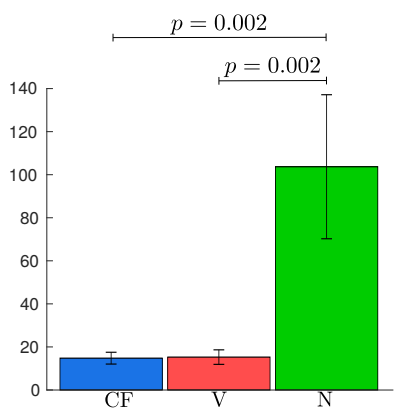

(b) Translation error ( $\mathrm{mm})$

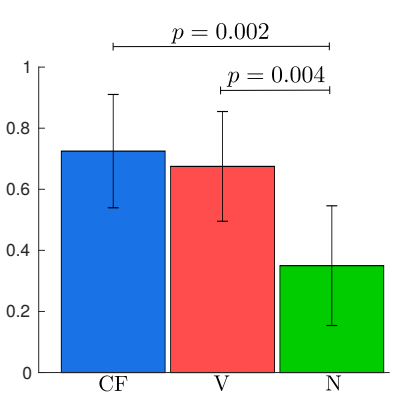

(c) Success rate

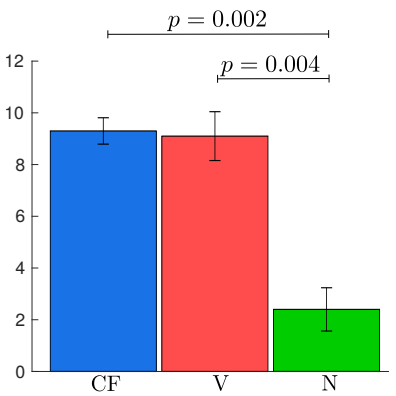

(d) Perceived effectiveness

Fig. 7. Experiment \#2. RMS orientation and position error, success rate, and perceived effectiveness provided a measure of performance for conditions CF (in blue), V (in red), and $\mathrm{N}$ (in green). Mean and $95 \%$ confidence interval are plotted.

and V vs. $\mathrm{N}(\mathrm{p}=0.004)$. The failures were due to too high collisions forces between the robot and the obstacle, which caused the manipulator to go into protective mode. We also measured the completion time. All the data passed the ShapiroWilk normality test and Mauchly's Test of Sphericity. A one-way repeated-measures ANOVA indicated no significant difference in the performance among feedback conditions. Average completion time was $168.93 \pm 10.52 \mathrm{~s}$ (mean \pm SD). We also tested whether the task completion time affected the task performance. Our hypothesis is that subjects who took more time were able to also achieve higher performance in the considered metrics. A Pearson's product-moment correlation was run to assess the relationship between this distance and the error in following the desired trajectory with the gripper. A preliminary analysis showed that the relationship was linear with normally distributed variable, according to the ShapiroWilk test, and there were no outliers. There was a strong positive correlation between the completion time vs. rotation error $(\mathrm{r}(\mathrm{x})=0.750, p<0.001)$ and translation error $(\mathrm{r}(\mathrm{x})=$ $0.880, p<0.001)$, confirming our hypothesis.

Besides this evaluation, we also assessed the users' experience. Immediately after the experiment, subjects were asked to complete a questionaire that used bipolar Likerttype eleven-point scales, reporting the effectiveness of each feedback condition in completing the given task. Figure $7 d$ shows the perceived effectiveness of the three feedback conditions. A Friedman test showed a statistically significant difference between the means of the feedback conditions $\left(\chi^{2}(2)=16.270, p<0.001\right)$. Post hoc analysis with Bonferroni correction revealed a statistically significant difference between conditions CF vs. N (p = 0.002) and V vs. N (p $=0.004)$. Finally, subjects were also asked to choose the condition they preferred the most. Condition $\mathrm{CF}$ was preferred by five subjects and condition $\mathrm{V}$ was preferred by five subjects. No subject reported any significant change in the fastening force or in the positioning of the device during the task.

\section{Discussion AND CONCLUSIONS}

In this paper we presented a wearable cutaneous device able to provide navigation cues through lateral skin stretch haptic feedback. The device is shown in Figs. 1. Four rotating end effectors with cylindrical shape can generate skin stretches independently at the ulnar, radial, palmar, and dorsal sides of a user's forearm. When the end effectors all rotate in the same direction, the device provides cutaneous cues about a desired pronation/supination of the forearm. When two opposite end effectors rotate in opposite directions, the device provides cutaneous cues about a desired translation of the forearm. The device weights $95 \mathrm{~g}$, it adds only $2.5 \mathrm{~cm}$ to the wrist size, and it is wireless. A separate arm band, weighting $280 \mathrm{~g}$, provides the required power and electronics.

We carried out two experiments of haptic navigation, enrolling a total of 20 subjects. In the first experiment, subjects had to translate and rotate the forearm toward a target position and orientation, respectively. Our haptic device (CF) and sensory substitution with visual feedback (V) significantly improved the performance of the task with respect to providing no navigation feedback (N). Sensory substitution with visual feedback outperformed cutaneous feedback in one metric. Finally, most subjects preferred visual feedback over cutaneous feedback, as they found the visual information more natural and requiring less concentration with respect to the cutaneous one. Nonetheless, this large difference in preference ( 8 vs. 2 subjects for condition V) did not reflect the task performance. In fact, a significant lower error of condition $\mathrm{V}$ vs. condition $\mathrm{CF}$ was found only for the translation metric (see Fig. 4b), while in the rotation metric condition $\mathrm{CF}$ performed even better than condition $\mathrm{V}$ (although no statistically significant difference was found, see Fig. 4a).

In the second experiment, subjects were asked to control the motion and orientation of a 6-DoF robotic manipulator to grasp and lift a target object. Conversely to Experiment \#1, this time condition CF was more appreciated by the subjects, who considered it as effective as visual feedback. However, subjects again reported the visual condition to be more natural with respect to the cutaneous one. Nonetheless, 9 subjects out of 10 reported that in condition $\mathrm{V}$ it was hard to concentrate, at the same time, on the arrow indicating the target hand pose and on the camera view of the remote environment (see top of Fig. 5a). In this respect, providing navigation information through the skin stretch device prevented an overload of the visual channel. Similar results have been demonstrated also for other teleoperation applications, where providing 
cutaneous feedback outperformed sensory substitution via visual cues [28]-[31]. Results also showed that cutaneous feedback and sensory substitution with visual feedback significantly improved the performance of the task with respect to providing no navigation feedback. No significant difference was found between conditions CF and V.

Our skin stretch device was proven to be a viable solution for navigation feedback in human guidance and robotic telemanipulation systems. However, haptic guidance was still found to be less natural to follow than visual guidance. In this respect, we should take into account that we are used everyday to follow visual navigation cues (e.g., turn-by-turn car navigation systems, road signs), while we are not used to follow haptic navigation stimuli at all. In this respect, we expect more training to improve the performance of the haptic modality. Moreover, while visual cues may be superior when there are no other cues to follow, whenever the operator receives more than one information flow through the visual channel, providing haptic feedback seems a very good option. This is a common situation in robotic teleoperation, where the human operator looks at the camera visual stream and, at the same time, is provided with additional information about the forces exerted by the slave robot, a predetermined trajectory to follow, or the presence of surrounding obstacles. While these pieces of information can be superimposed on the screen, as we have done in condition $\mathrm{V}$ of Experiment \#2, our results show that using haptic feedback can provide comparable performance without overloading the visual channel - proving cutaneous feedback to be the best choice.

In the future, we plan to run more experiments to study how the performance and subjects' preference change after some training. We also plan to test the effect of providing navigation information using visual and skin stretch cues together. Finally, we also plan to run a longer evaluation session, in order to understand if the current fasting mechanism is capable of keeping the device in place during a prolonged use.

\section{REFERENCES}

[1] C. Pacchierotti, S. Sinclair, M. Solazzi, A. Frisoli, V. Hayward, and D. Prattichizzo, "Wearable haptic systems for the fingertip and the hand: taxonomy, review, and perspectives," IEEE Trans. Haptics, 2017.

[2] F. Chinello, C. Pacchierotti, M. Malvezzi, and D. Prattichizzo, "A three revolute-revolute-spherical wearable fingertip cutaneous device for stiffness rendering," IEEE Trans. Haptics, 2017.

[3] C. Pacchierotti, Cutaneous haptic feedback in robotic teleoperation, ser. Springer Series on Touch and Haptic Systems, 2015.

[4] Z. F. Quek, S. B. Schorr, I. Nisky, A. M. Okamura, and W. R. Provancher, "Sensory augmentation of stiffness using fingerpad skin stretch," in Proc. IEEE World Haptics, 2013, pp. 467-472.

[5] — , "Augmentation of stiffness perception with a 1-degree-of-freedom skin stretch device," IEEE Trans. Human-Machine Systems, vol. 44, no. 6, pp. 731-742, 2014.

[6] L. Meli, C. Pacchierotti, and D. Prattichizzo, "Experimental evaluation of magnified haptic feedback for robot-assisted needle insertion and palpation," International Journal of Medical Robotics and Computer Assisted Surgery, 2017.

[7] C. Pacchierotti, A. Tirmizi, G. Bianchini, and D. Prattichizzo, "Enhancing the performance of passive teleoperation systems via cutaneous feedback," IEEE Trans. Haptics, vol. 8, no. 4, pp. 397-409, 2015.

[8] C. Pacchierotti, L. Meli, F. Chinello, M. Malvezzi, and D. Prattichizzo, "Cutaneous haptic feedback to ensure the stability of robotic teleoperation systems," International Journal of Robotics Research, vol. 34, no. 14, pp. 1773-1787, 2015.
[9] A. Spiers and A. Dollar, "Design and evaluation of shape-changing haptic interfaces for pedestrian navigation assistance," IEEE Trans. Haptics, 2016.

[10] Y. Imamura, H. Arakawa, S. Kamuro, K. Minamizawa, and S. Tachi, "Hapmap: Haptic walking navigation system with support by the sense of handrail," in Proc. ACM SIGGRAPH - Emerging Tech., 2011, p. 6.

[11] F. Hemmert, S. Hamann, M. Löwe, J. Zeipelt, and G. Joost, "Shapechanging mobiles: tapering in two-dimensional deformational displays in mobile phones," in Proc. CHI, 2010, pp. 3075-3080.

[12] T. Oron-Gilad, J. L. Downs, R. D. Gilson, and P. A. Hancock, "Vibrotactile guidance cues for target acquisition," IEEE Trans. Systems, Man, and Cybernetics, Part C, vol. 37, no. 5, pp. 993-1004, 2007.

[13] J. Rekimoto, "Traxion: a tactile interaction device with virtual force sensation," in Proc. ACM SIGGRAPH - Emerging Tech., 2014, p. 25.

[14] B. T. Gleeson, S. K. Horschel, and W. R. Provancher, "Design of a fingertip-mounted tactile display with tangential skin displacement feedback," IEEE Trans. Haptics, vol. 3, no. 4, pp. 297-301, 2010.

[15] M. Maisto, C. Pacchierotti, F. Chinello, G. Salvietti, A. De Luca, and D. Prattichizzo, "Evaluation of wearable haptic systems for the fingers in augmented reality applications," IEEE Trans. Haptics, 2017.

[16] A. Stanley, K. J. Kuchenbecker et al., "Evaluation of tactile feedback methods for wrist rotation guidance," IEEE Trans. Haptics, vol. 5, no. 3, pp. 240-251, 2012.

[17] M. F. Rotella, K. Guerin, X. He, and A. M. Okamura, "Hapi bands: a haptic augmented posture interface," in IEEE Haptics Symposium (HAPTICS), 2012, pp. 163-170.

[18] V. Yem, M. Otsuki, and H. Kuzuoka, "Development of wearable outercovering haptic display using ball effector for hand motion guidance," in Haptic Interaction, 2015, pp. 85-89.

[19] T. Amemiya and H. Sugiyama, "Orienting kinesthetically: A haptic handheld wayfinder for people with visual impairments," ACM Trans. Accessible Computing, vol. 3, no. 2, p. 6, 2010.

[20] Y. Kuniyasu, M. Sato, S. Fukushima, and H. Kajimoto, "Transmission of forearm motion by tangential deformation of the skin," in Proc. 3rd Augmented Human International Conference, 2012, p. 16.

[21] R. L. Koslover, B. T. Gleeson, J. T. de Bever, and W. R. Provancher, "Mobile navigation using haptic, audio, and visual direction cues with a handheld test platform," IEEE Trans. Haptics, vol. 5, no. 1, pp. 33-38, 2012.

[22] T. Nakamura, N. Nishimura, M. Sato, and H. Kajimoto, "Development of a wrist-twisting haptic display using the hanger reflex," in Proc. Conf. Advances in Computer Entertainment Technology, 2014, p. 33.

[23] F. Chinello, C. Pacchierotti, N. G. Tsagarakis, and D. Prattichizzo, "Design of a wearable skin stretch cutaneous device for the upper limb," in Proc. IEEE Haptics Symposium (HAPTICS), 2016, pp. 14-20.

[24] P. t. Burgess and E. Perl, "Cutaneous mechanoreceptors and nociceptors," in Somatosensory system. Springer, 1973, pp. 29-78.

[25] J. Bimbo, C. Pacchierotti, M. Aggravi, N. G. Tsagarakis, and D. Prattichizzo, "Teleoperation in cluttered environments with distributed wearable haptic feedback," in Proc. IEEE/RSJ Int. Conf. Intelligent Robots and Systems, 2017.

[26] M. G. Catalano, G. Grioli, E. Farnioli, A. Serio, C. Piazza, and A. Bicchi, "Adaptive synergies for the design and control of the pisa/iit softhand," Int. J. Robot. Res., vol. 33, no. 5, pp. 768-782, 2014.

[27] E. Ostergaard, "Lightweight robot for everybody [industrial activities]," IEEE Robot. Autom. Mag., vol. 19, no. 4, pp. 17-18, 2012.

[28] C. Pacchierotti, D. Prattichizzo, and K. J. Kuchenbecker, "Cutaneous feedback of fingertip deformation and vibration for palpation in robotic surgery," IEEE Trans. Biomed. Eng., vol. 63, no. 2, pp. 278-287, 2016.

[29] M. Abayazid, C. Pacchierotti, P. Moreira, R. Alterovitz, D. Prattichizzo, and S. Misra, "Experimental evaluation of co-manipulated ultrasoundguided flexible needle steering," Int J Med Robot, vol. 12, no. 2, pp. 219-230, 2016.

[30] D. Prattichizzo, C. Pacchierotti, and G. Rosati, "Cutaneous force feedback as a sensory subtraction technique in haptics," IEEE Trans. Haptics, vol. 5, no. 4, pp. 289-300, 2012.

[31] C. Pacchierotti, M. Abayazid, S. Misra, and D. Prattichizzo, "Teleoperation of steerable flexible needles by combining kinesthetic and vibratory feedback," IEEE Trans. Haptics, vol. 7, no. 4, pp. 551-556, 2014. 